\title{
THE EFFECTS OF BENZODIAZEPINE (VALIUM) ON PERMEABILITY OF LANGUAGE EGO BOUNDARIES ${ }^{1}$
}

\author{
Alexander Z. Guiora, William R. Acton, ${ }^{2}$ Robert Erard, and \\ Fred W. Strickland, Jr. \\ The University of Michigan
}

\begin{abstract}
This study was designed as an extension of previous research in which pronunciation of a foreign language was experimentally manipulated using alcohol or hypnosis, to confirm a relationship between permeability of language ego boundaries and pronunciation. The present study used Benzodiazepine (Valium) to manipulate pronunciation in Thai. Seventy-five subjects were assigned to one of four treatment conditions: Placebo, $2 \mathrm{mg}$., $5 \mathrm{mg}$., or $10 \mathrm{mg}$, and subsequently tested on the Standard Thai Procedure (STP) and the Digit Symbol Test. Results seem to suggest that Benzodiazepine (Valium) facilitates the empathic sensitivity of the subjects to the tester rather than to the voice on the tape. The combined findings of the Valium and alcohol studies are interpreted as supporting the theoretical connection between language ego boundaries and ego boundaries in general and illustrate the extraordinary sensitivity of the test to fluctuations in the state of the subject's ego.
\end{abstract}

In successive publications (Guiora 1967, Guiora 1970, Guiora 1972, Guiora et al. 1968, Guiora et al. 1972, Taylor et al. 1969) we developed the hypothesis that individual variation in the ability to approximate nativelike pronunciation in a second language is, in part, determined by certain psychological variables best subsumed under the construct empathy or, more broadly, the concept of permeability of ego boundaries. These variables have been investigated in three manifestations: (1) as stable characteristics of adults, (2) as milestones in the growth curve in the course of development, and (3) as experimentally induced behaviors.

In our program dealing with the third of these propositions, i.e., the experimental induction of behaviors, we had planned to study the effects of different agents (e.g., alcohol, drugs, hypnosis) on the target behavior (i.e.,

\footnotetext{
1 We gratefully acknowledge the invaluable counsel of J.E.K. Smith-so generously extended-in matters of data analysis. We are indebted to Allison Edwards, Dobroslaw Lachowicz, James Runner, and Douglas Woken for their assistance in running the experiment. Stephen Tyma has read an earlier version of this paper, and we wish to thank him for his comments.

This study was supported in part by The University of Michigan Medical School's Biomedical Research Grant No. RR 05383.

${ }^{2}$ Dr. Acton is now on the faculty of the University of Houston.
} 
pronunciation). One such study completed some time ago attempted to achieve lowering of inhibitions or enhancing of the permeability of ego boundaries by means of controlled ingestion of alcohol (Guiora et al. 1972).

"Lowering of inhibitions" (or behaviors described as such) may be conceptualized as the result of an alteration of particular, internal processes. For example, mild vertigo, due to alcohol consumption can be seen as stemming from a reduced efficiency in the functions regulating equilibrium. Similarly, slips of the tongue which often occur after alcohol consumption, appear to be related to changes in "intrapsychic equilibrium," i.e., certain thoughts which were previously kept out of conscious awareness suddenly and involuntarily find a linguistic outlet.

These examples are but two specific illustrations of the myriad of internal processes called "ego functions." Some others include eye-hand coordination, accurate perception of reality, logical thought, etc. A much more complex ego function, whose fluctuations are not as easily recognized in specific behaviors as those mentioned above, is the ability to maintain one's identity separate from others. It has been hypothesized that the capacity to partially and temporarily give up the "separateness" is largely responsible for the phenomenon known as empathy (Guiora 1972).

The "alcohol" study focused on the effects of alcohol consumption upon the ability to partially and temporarily give up this separateness of identity. It was hypothesized that one manifestation of this ability (conceptualized as permeability of ego boundaries) is an increased capacity to pronounce a foreign language authentically.

The idea that certain inhibitions are lowered by drugs (including alcohol) is widespread and properly documented. Disinhibited behavior in turn can be seen as a manifestation, a concomitant of underlying psychological processes, of a higher level construct, i.e., permeability of ego boundaries. It is not unreasonable to assume that by manipulating the behavioral correlate (disinhibited behavior) one can have an effect on the postulated high construct (permeability of ego boundaries). If this is so, then the induction of disinhibited behavior can be seen as manipulation of the underlying processes themselves.

\section{The Alcohol Study}

The purpose of the study was to examine the effects of a small amount of alcohol on the ability of college students to pronounce words and phrases in a foreign and totally unknown language (Thai). We hypothesized that the effect 
would be an improvement in the ability to approximate authentic pronunciation as compared to a control group who would not consume alcohol but a placebo designed to simulate alcohol.

Eighty-seven University of Michigan students served as subjects. All were over 21 years of age and were informed in advance only that the experiment would involve responses to an alcoholic beverage. The results of this study are highly illuminating. The hypothesis that the experimentally induced lowering of inhibitions or enhancing of the permeability of ego boundaries will lead to a corresponding enhancement of the pronunciation flexibility was conclusively confirmed $(\mathrm{t}=4.47, \mathrm{p} \leqslant 0.01)$.

This finding is all the more interesting in light of the fact that overall mental functioning (cognitive, psychomotor, memory, i.e., integrated ego functioning), as measured by the Digit Symbol Test, is not adversely affected by the same condition. In other words, pronunciation "permeability" is apparently critically influenced by a psychological variable that can be successfully isolated out of the total web of ego functioning.

\section{The Hypnosis Study}

In a recent study, Schumann, Holroyd, Campbell, and Ward (1978), following Guiora's suggestion (Guiora 1972), tested the hypothesis that "improvement in pronunciation ability will be greater for high hypnotizable than for low hypnotizable subjects." They report that "when groups were defined in terms of their own ratings, deeply hypnotized subjects performed significantly better than less hypnotized subjects $(\mathrm{F}=5.21, \mathrm{df}=2, \mathrm{p}<.01)$." They conclude that "the results are consistent with both Guiora's line of reasoning about permeability of ego boundaries and Hilgard's neodissociation theory."

\section{Present Study}

As a logical extension of these studies we wished to examine the positive effects a chemical relaxant might have on the enhancement of pronunciation ability and, by inference, on the construct under study, the permeability of ego boundaries. Because Benzodiazepines are well known for their capacity to reduce anxiety with minimal adverse side effects, we chose Valium as our experimental agent.

In assessing the merits of various routes of administration of Benzodiazepines for the purposes of this study, parenteral administration was deemed 
inadvisable because it was felt that the anxiety which would accompany an injection would, for some subjects, significantly influence their subsequent performance on the Standard Thai Procedure (STP) and Digit Symbol Test, thus introducing an unacceptable source of error into the experiment. In addition, it was noted that oral administration of Benzodiazepines has been shown superior to intramuscular administration in insuring rapid and reliable absorption (Greenblatt et al. 1974, Hillestad et al. 1974, Kanto 1975, Shader and Greenblatt 1977, Gottschalk et al. 1974), while intravenous administration involves some risk of complications (Greenblatt and Koch-Weser 1973, Hegarty and Dundee 1977). Finally, it was noted that use of an oral Benzodiazepine preparation would permit a wider range of choice in regard to the specific agent to be employed.

The experimental design also required, in the interest of increased subject compliance, that the STP and Digit Symbol Test be given within approximately 45 minutes of Benzodiazepine administration. It was therefore necessary that the specific Benzodiazepine chosen exert its clinical effect within this time period. Orally administered Valium has generally been shown to produce a clinical effect in less than 60 minutes (Besser and Duncan 1967, Bliding 1974, Klotz et al. 1975), in contrast to other Benzodiazepines (Bliding 1974, Boxenbaum et al. 1977, Greenblatt et al. 1977).

In addition, the clinical effects of Benzodiazepines appear to be related to the concentration of the drug within the blood (Hillestad et al. 1974, Kanto 1975 ) or to the rate of change of blood levels (Bliding 1974, Greenblatt et al. 1977). Again, orally administered Valium has been found to rise to its peak blood levels within the desired period (Kaplan et al. 1973, Bliding 1974, Dasberg 1974, Greenblatt et al. 1974, Gamble et al. 1975, Hillestad et al. 1974, Kanto 1975, Wretlind et al. 1977), in contrast to other orally administered Benzodiazepines which reached their peak blood levels more slowly (Gottschalk et al. 1974, Greenblatt et al. 1976, Kaplan et al. 1976, Elliott 1976, Greenblatt et al. 1977). Thus, Valium was chosen as the specific Benzodiazepine to be used in this study.

It should be noted, however, that Valium may show significant variation (as much as seven-fold) in peak blood levels from one individual to another (Kaplan et al. 1974, Bliding 1974, Gamble et al. 1975, Hillestad et al. 1974, Klotz et al. 1975, Wretlind et al. 1977). The speed with which this peak is attained may also vary significantly (Kaplan et al. 1973), and other Benzodiazepines also show significant variation in peak blood levels (Schwartz et al. 1971, Bliding 1974, Greenblatt et al. 1974, Wretlind et al. 1977, Shader et al. 1977). 
It was anticipated that the clinical effect of a given dose of Valium would likewise vary significantly from one individual to another, thus introducing the possibility of significant variation in the observed behavior. This difficulty could have been circumvented by measuring blood levels at the time that the STP and Digit Symbol Tests were given; however, as noted in the above discussion of parenteral administration, it was felt that venipuncture to assess blood levels would be accompanied by anxiety that would introduce an unacceptable degree of behavioral perturbation. It was therefore decided to forego measurements of blood levels of Valium, and to seek instead to minimize individual variations in blood levels of Valium by recruiting a population of subjects homogeneous in regard to factors known to influence blood levels of Valium.

Such factors include age (Klotz et al. 1975), a history of use of sedative hypnotic drugs (Kanto et al. 1974), a history of ethanol use (Sellman et al. 1975), antacids or anti-cholinergic medications (Shader and Greenblatt 1977, Greenblatt et al. 1977, Gamble et al. 1976), and weight (Gamble et al. 1975). Prospective subjects were thus evaluated in regard to each of these factors by means of a screening questionnaire.

\section{Method}

Subjects for the experimental conditions were obtained through an advertisement in the local student newspaper and through the Human Performance Center Subject Pool at the University of Michigan. Subjects who were to receive Valium or a placebo in the experiment were told in advance that as part of the experiment they would be given a small, undisclosed amount of Valium. Subjects for the No-drug control condition were unaware of the use of Valium in the experiment and were tested separately.

All Ss were screened for any familiarity with the Thai language or any formal training in phonetics. Ss in the drug condition pool were additionally screened for any medical contraindications and each gave informed consent to receive an undisclosed amount of Valium up to 10 milligrams. These subjects were randomly assigned to one of four treatment conditions: placebo, $2 \mathrm{mg}$., $5 \mathrm{mg}$, , or $10 \mathrm{mg}$. The experiment was run in the morning and all subjects were given a light breakfast of pastries and orange juice to make blood levels more uniform. Drugs were administered in a double-blind arrangement 45 minutes prior to testing. The No-drug group was tested separately.

Each subject was individually administered the STP (see Appendix) and 
the Digit Symbol Test (a subtest of the Wechsler Adult Intelligence Scale which serves as a rough measure of psychomotor coordination) by one of three testers in a separate room. Following testing, all Ss were debriefed on the nature of the study, were advised of the amount of Valium they had each received and its probable effects, and were paid five dollars.

Due to a no-show rate of about twenty-five percent among Ss who originally agreed to participate in the study, the experiment was repeated with a smaller group of Ss on a separate occasion to replace lost subjects. Unfortunately, one of the original testers was unavailable for the second run and had to be replaced. At the time, this seemed to be a trivial matter since the tester's function was limited to operating the tape machine for the STP, reading a standard set of instructions for the Digit Symbol Test, and announcing when the time limit was up on the latter test.

Seventy-five subjects in all were tested. The STP tapes were each scored independently by three trained native Thai speakers. Interrater reliability ranged from .75 to .85 . Final scores for each subject were determined by averaging the scores of each of the three raters.

\section{Results}

Contrary to expectation, the differences in the mean STP scores among treatment groups were unsystematic and nonsignificant. However, the variance of the scores increased monotonically as the drug dosage increased. The correlation is .98 ; this result is significant at the .01 level.

Further inspection of the data revealed that STP mean scores varied significantly according to which tester presented the STP to the subject $(\mathrm{F}=4.97, \mathrm{df}=3.71, \mathrm{p}=.01)$. The increase in variance in STP score as dosage increased was highly correlated with the variance among testers' groups. That is, as dosage increased, the effect of having been tested by a particular person also increased, and this seems to account for the increasing variance among scores as dosage increased.

A two-way analysis of variance involving all drug conditions and the three testers who had subjects in every condition showed a significant interaction $(p=.05)$ between tester and drug condition.

It seems to be clear that a subject's score is significantly influenced by the individual who does the testing and that this susceptibility to influence becomes more powerful as Valium dosage increases. Neither Valium dosage nor tester had significant effects on the Digit Symbol Test. 
Table 1

\begin{tabular}{cccc}
\hline Mg. Valium & $\begin{array}{c}\text { STP standard } \\
\text { deviation }\end{array}$ & $\begin{array}{c}\text { STP } \\
\text { variance }\end{array}$ & $\begin{array}{l}\text { Mean square } \\
\text { between tester } \\
\text { groups }\end{array}$ \\
\hline & & & \\
0 & 18.18 & 330.51 & 59.9 \\
2 & 21.76 & 472.50 & 1156.6 \\
5 & 27.72 & 768.40 & 1649.0 \\
10 & 40.05 & 1604.00 & 4820.2 \\
\hline
\end{tabular}

Table 2

\begin{tabular}{lccccc}
\hline & Sum of squares & D.F. & Mean square & F & P \\
\hline Tester & 5631.24 & 2 & 2815.62 & 4.46 & .01 \\
Valium dosage & 214.74 & 4 & 53.69 & .09 & N.S. \\
Interaction & 11204.22 & 8 & 1400.53 & 2.22 & .05 \\
Error & 33422.51 & 53 & 630.61 & & \\
\hline
\end{tabular}




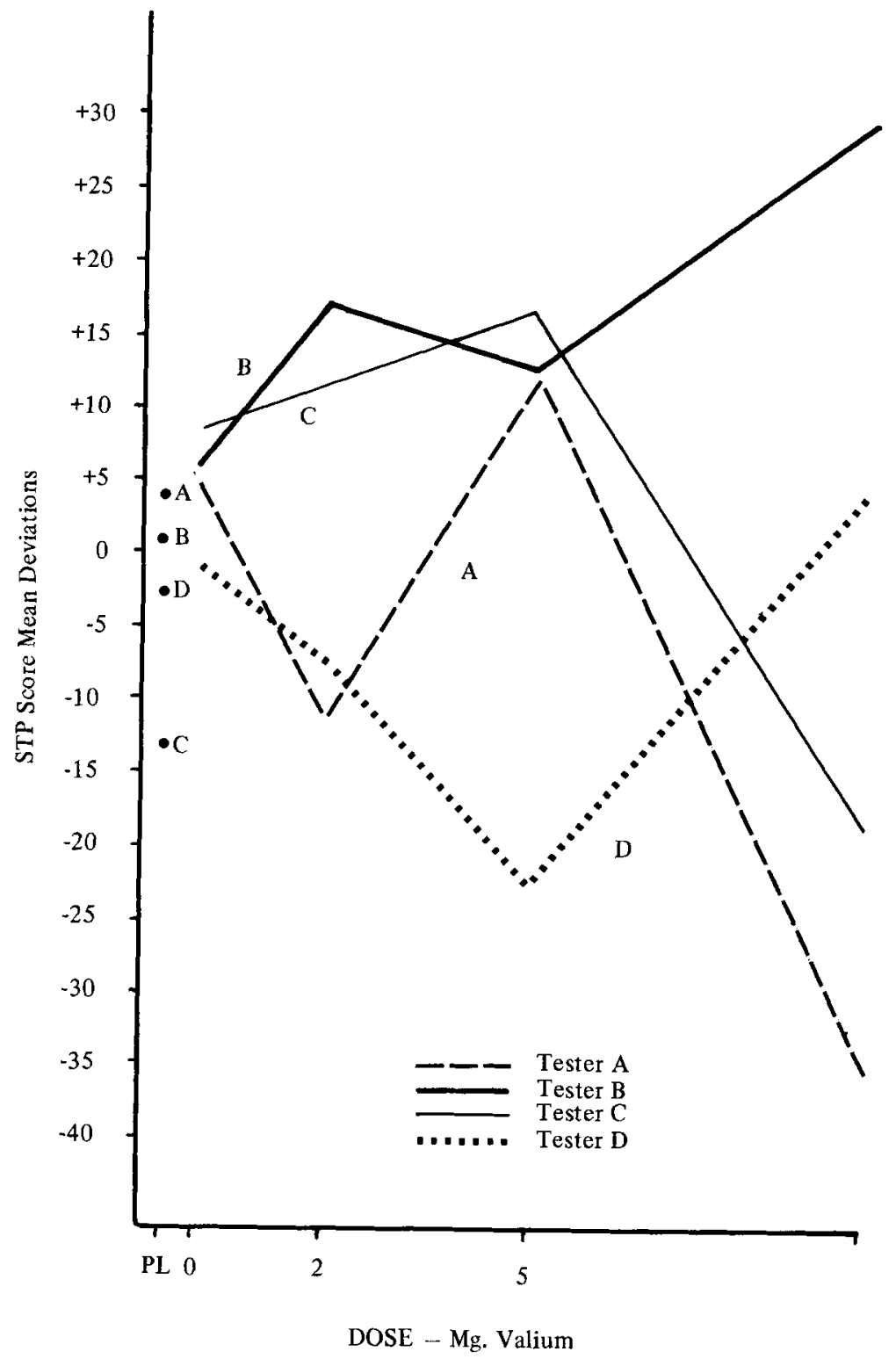

Figure 1 


\section{Discussion}

Our initial hypothesis, that performance on the STP would be enhanced by Valium was not directly substantiated. This may be due to the fact that the effects of a specific dosage vary considerably according to the constitutional idiosyncrasies of the subject who receives it, more so than alcohol. To some extent this problem might have been controlled by determining the blood levels of Valium in each subject, but we felt that doing so would probably have engendered considerable anxiety and inhibition in our subjects, thus undermining our intent in using Valium in the first place.

The significant effect of tester on STP scores was quite surprising given the very limited and standardized role of the tester. However, informal reports from the testers indicate that the subjects did appear to be very sensitive to subtle cues such as eye contact and facial expression. Subjects may thus have been able to interpret nonverbal cues from the testers, placing them in the total context of the interaction, and reacting to them in their test behavior. In other words, the individual subject's test behavior was apparently influenced (positively or negatively) by the "vibes" he or she perceived as coming from the tester, and this "susceptibility to influence" increased with Valium dosage.

Thus, the fact that there was a significant interaction between dosage and tester effect suggests that Valium does affect permeability of ego boundaries, but in a different way than we had anticipated. Valium seems to facilitate the empathic sensitivity of the subjects not to the voice on the tape but to the more immediate and salient stimulus of the testers themselves. The combined findings of the Valium and alcohol studies support the theoretical connection between language ego boundaries and ego boundaries in general and illustrate the extraordinary sensitivity of the test to fluctuations in the state of the subject's ego.

\section{REFERENCES}

Besser, G.M., and C. Duncan. 1967. The time course of action of single doses of diazepam, chlorpromazine and some barbiturates as measured by auditory flutter fusion and visual flicker fusion thresholds in man. British Journal of Pharmacology and Chemotherapy 30:341-348.

Bliding, A. 1974. Effects of different rates of absorption of two benzodiazepines on subjective and objective parameters. European Journal of Clinical Pharmacology $7: 201-211$.

Boxenbaum, H.G., K.A. Geitner, M.L. Jack, W.R. Dixon, H.E. Spiegel, J. Symington, 
R. Christian, J.D. Moore, L. Weissman, and S.A. Kaplan. 1977. Pharmacokinetic and biopharmaceutic profile of chlordiazepoxide $\mathrm{HCl}$ in healthy subjects: single-dose studies by the intravenous, intramuscular, and oral routes. Journal of Pharmacokinetics and Biopharmaceutics 5(1):3-23.

Dasberg, H. 1974. The effect of daily oral dosage of diazepam, plasma concentrations and metabolic clearance of diazepam and desmethyldiazepam on various constituents of the acute clinical anxiety syndrome. Psychotherapy and Psychosomatics $24: 113$ 118.

Elliott, H.W. 1976. Metabolism of lorazepam. British Joumal of Anaesthesia 48(10): 1017-1023.

Gamble, J.A., J.W. Dundee, and R.A. Assaf. 1975. Plasma diazepam levels after single dose oral and intramuscular administration. Anaesthesia 30(2):164-169.

Gamble, J.A., J.H. Gaston, S.G. Nair, and J.W. Dundee. 1976. Some pharmacological factors influencing the absorption of diazepam following oral administration. British Journal of Anaesthesia 48(12):1181-1185.

Gottschalk, L.A., R. Biener, and E.C. Dinovo. 1974. Effect of oral and intramuscular routes of administration on serum chlordiazepoxide levels and the prediction of these levels from predrug fasting serum glucose concentrations. Research Communications in Chemical Pathology and Pharmacology 8(4):697-702.

Greenblatt, D.J., and J. Koch-Weser. 1973. Adverse reactions to intravenous diazepam: a report from the Boston Collaborative Drug Surveillance Program. American Journal of Medical Science 266:261-266.

Greenblatt, D.J., R.T. Schillings, A.A. Kyriakopoulos, R.I. Shader, S.F. Sisenwine, J.A. Knowles, and H.W. Ruelius. 1976. Clinical pharmacokinetics of lorazepam. I. Absorption and disposition of oral 14C-lorazepam. Clinical Pharmacology and Therapeutics 20(3):329-341.

Greenblatt, D.J., R.I. Shader, J.S. Harmatz, K. Franke, and J. Koch-Weser. 1977. Absorption rate, blood concentrations, and early response to oral chlordiazepoxide. American Journal of Psychiatry 134(5):559-562.

Greenblatt, D.J., R.I. Shader, and J. Koch-Weser. 1974. Pharmacokinetic determinants of the response to single doses of chlordiazepoxide. American Journal of Psychiatry 131(12): 1395-1397.

Greenblatt, D.J., R.I. Shader, and J. Koch-Weser. 1974. Slow absorption of intramuscular chlordiazepoxide. New England Joumal of Medicine 291(21):1116-1118.

Guiora, A.Z. 1967. Toward a systematic study of empathy. Comprehensive Psychiatry $8: 5$.

Guiora, A.Z. 1970. Transpositional research in the clinical process. Comprehensive Psychiatry 11:6.

Guiora, A.Z. 1972. Construct validity and transpositional research, toward an empirical study of psychoanalytic concepts. Comprehensive Psychiatry 13:139-150.

Guiora, A.Z., B. Beit-Hallahmi, R.C.L. Brannon, C.Y. Dull, and T. Scovel. 1972. The effects of experimentally induced changes in ego states on pronunciation ability in a second language: an exploratory study. Comprehensive Psychiatry 13:421-428.

Guiora, A.Z., H.L. Lane, and L.A. Bosworth. 1968. An exploration of some personality variables in authentic pronunciation of a second language. In Lane, H.L., and E.M. Zale (eds.), Studies in Language and Language Behavior, 463-474.

Hegarty, J.R., and J.W. Dundee. 1977. Sequelae after the intravenous injection of three benzodiazepines-diazepam, lorazepam, and flunitrazepam. British Medical Journal 2:1384-1385,

Hillestad, L., T. Hansen, H. Melsom, and A. Drivenes. 1974. Diazepam metabolism 
in normal man I. Serum concentrations and clinical effects after intravenous, intramuscular, and oral administration. Clinical Pharmacology and Therapeutics 16(3): 479-484.

Kanto, J. 1975. Plasma concentrations of diazepam and its metabolites after peroral, intramuscular, and rectal administration. International Joumal of Clinical Pharmacology 12(4): 427-432.

Kanto, J., E. Iisalo, V. Lehtinen, and J. Salminen. 1974. The concentrations of diazepam and its metabolites in the plasma after an acute and chronic administration. Psychopharmacologia (Berlin) 36:123-131.

Kaplan, S.A., M.L. Jack, K. Alexander. and R.E. Weinfeld. 1973. Pharmacokinetic profile of diazepam in man following single intravenous and oral and chronic oral administrations. Journal of Pharmaceutical Sciences 62:1789-1796.

Kaplan, S.A., M.L. Jack, R.E. Weinfeld., W. Glover, L. Weissman, and S. Cotler. 1976. Biopharmaceutical and clinical pharmacokinetic profile of bromazepam. Journal of Pharmacokinetics and Biopharmaceutics 4(1):1-16.

Klotz, U., G.R. Avant, A. Hoyumpa, S. Schenker, and G.R. Wilkinson. 1975. The effects of age and liver disease on the disposition and elimination of diazepam in adult man. Joumal of Clinical Investigation 55(2):347-359.

Schumann, J.H., J. Holroyd, R.N. Campbell, and F.A. Ward. 1978. Improvement of foreign language pronunciation under hypnosis. Language Learning 28:1.

Schwartz, M.A., E. Postma, and Z. Gaut. 1971. Biological half-life of chlordiazepoxide and its metabolite, demoxepam, in man. Journal of Pharmaceutical Sciences 60: 1500-1503.

Sellman, R., A. Pekkarinen, L. Kangas, and E. Raijola. 1975. Reduced concentrations of plasma diazepam in chronic alcohoiic patients following an oral administration of diazepam. Acta Pharmacologia et Toxocologia (Kbh) 36(2):25-32.

Shader, R.I., and D.J. Greenblatt. 1977. Clinical implications of benzodiazepine pharmacokinetics. American Journal of Psychiatry 134(6):652-656.

Shader, R.I., D.J. Greenblatt, J.S. Harmatz, K. Franke, and J. Koch-Weser. 1977. Absorption and disposition of chlordiazepoxide in young and elderly male volunteers. Journal of Clinical Pharmacology 17(11-12):709-718.

Taylor, L.L., A.Z. Guiora, J.C. Catford, and H.L. Lane. 1969. The role of personality variables in second language behavior. Comprehensive Psychiatry 10:463-474.

Wretlind, M., A. Pilbrant, A. Sundwall, and J. Vessman. 1977. Disposition of three benzodiazepines after single oral administration in man. Acta Pharmacologia et Toxicologia (suppl) (Kbh) 40(1):28-39. 


\section{APPENDIX \\ The Standard Thai Procedure (STP)}

The STP was developed for the purpose of assessing second language pronunciation ability and to be used uniformly in all our studies. The major concern in constructing the test was that of appropriateness of use with almost any population (regardless of age or education, etc.). It was felt that the best language should be one not commonly taught or studied in this country. In addition, it seemed important that the language be relatively easy to pronounce for English speaking subjects, yet at the same time relatively distant, phonetically, from English. These considerations plus the practical constraint of availability of native speakers experienced in evaluating pronunciation led us eventually to choose the Thai language.

The test consists of ten one-syllable contrastive items and six expansion drill series. Each expansion drill series is composed of four items beginning with a single syllable to which a second, third, and fourth syllable are added successively. An example of such a series is as follows:

Diagrammatically: $\quad$ English Example:

$\begin{array}{ll}X & \text { since } \\ \mathrm{XX} & \text { since swifts } \\ \mathrm{XXX} & \text { since swifts pass } \\ \mathrm{XXXX} & \text { since swifts pass swans }\end{array}$

Six such expansion drill series generated 24 of the total 34 test items.

Evaluation: A total of 28 pronunciation units in the test are scored. Sixteen of these are elements consisting of a single syllable and are evaluated for one of the following features: tone, vowel length or combination, initial or final consonant. The remaining 12 items which are scored are the first and last syllables of the four-syllable utterances. Rating of these 12 syllables is general; that is, tone, vowel consonant quality are not isolated parameters as in the first 16 monosyllabic test items.

Scores for the STP are developed in the following manner. Each of the 28 pronunciation units is given a rating on a scale of 1-3 as described below. 
1. Poor - Strongly marked with foreign accent or almost unintelligible

2. Fair - Passable and not likely to cause any misunderstanding though not completely native-like

3. Good - Quite native-like or close to native-like pronunciation

Data tapes for all subjects are identified by code numbers and evaluated independently and simultaneously by a team of 3 experts in the Thai language. A subject's total raw score is the sum of each of the 3 rater's 28 evaluations; thus the maximum score is 252 points. Interjudge reliability on these ratings average $r=.81$; the homogeneity of total scores based on 3 raters (coefficient alpha) reached a highly acceptable 89 .

The ten contrastive items are all of one syllable. Five of these items test for the five Thai tones using the same syllable. Two of the remaining five items contrast aspiration and non-aspiration; two more contrast vowel length, and the last tests for initial velar nasal sound.

The 34 test items are presented in the following order: 16 one-syllable items, 6 two-syllable items, 6 three-syllable items, and 6 four-syllable items. It should be noted that while syllable difficulty increases with the progressive addition of syllables, there is a certain practice effect built into the test due to the expansion drill series. By the time the subject reaches the four-syllable items, only the fourth syllable is unrehearsed, while the first syllable has previously been uttered three times, the second syllable two times, and third syllable once.

Presentation: The STP is an aural-oral test; each of the 34 test items is recorded on a master tape. The voicer is a female native Thai speaker. Between each utterance there is a pause of about 4 seconds during which the testee is required to imitate the preceding utterance. A two-track recording is made during this test, with the stimuli recorded on one track and the subject's responses on the other. The total amount of time involved in administering this test is approximately $4 \frac{1}{2}$ minutes. The test tape is $2 \frac{1}{2}$ minutes long and instructions require about 2 minutes. 